\title{
Composición corporal por bioimpedancia eléctrica en estudiantes de escuelas de formación del Ejército colombiano
}

\author{
Jenner Cubides Amézquita \\ Esteban Aedo-Muñoz ${ }^{2}$ \\ Juan Camilo Mesa ${ }^{3}$ \\ Iván Dario Chavarro Castañeda
}

\section{Resumen}

Objetivo: el estudio hace una caracterización general de los parámetros bioeléctricos de la composición corporal de alumnos de último nivel de formación militar en tres escuelas de formación del Ejército Nacional de Colombia: Esmic, Emsub y Espro. Materiales y métodos: se planteó un estudio observacional de tipo transversal que midió cada una de las variables de la composición corporal y comparó los resultados entre los alumnos que conformaron la muestra. Para la medición de la composición corporal se usó el método indirecto de bioimpedancia eléctrica con el equipo SECA mBCA 525 (Medical Body Composition Analyzer, Hans E. Ruth S.A, Hamburg, Germany). Resultados: se encontró que las edades de los alumnos oscilaron entre los 18 a 25 años con una media de $21 \pm 1,5$ ańos, con un peso promedio de $66,3 \pm 7,9 \mathrm{~kg}$ y un Índice

1 Médico epidemiólogo. Grupo de Investigaciones Renfimil, Escuela Militar de Cadetes “General José María Córdova”, Bogotá, Colombia. Contacto: jenner.cubides@esmic.edu.co - https://orcid. org/0000-0001-6573-0432

2 PhD. en Ciencias de la Motricidad Humana. Laboratorio de Ciencias de la Actividad Física, el Deporte y la Salud, Universidad de Santiago de Chile, USACH, Chile. Contacto: esteban. aedo@usach.cl - https://orcid.org/0000-0003-1544-2824

3 Nutricionista Dietista. Pontificia Universidad Javeriana, Bogotá, Colombia. Contacto: juan. camilo.mesa.pimilla@stud.ki.se - https://orcid.org/0000-0002-6489-7609

4 Oficial de Artillería del Ejército Nacional de Colombia. Director del Centro de Investigaciones de la Cultura Física (Cicfi), Escuela Militar de Cadetes “General José María Córdova”, Bogotá, Colombia. Contacto: ivan.chavarroca@buzonejercito.mil.co - https://orcid.org/0000-0001-9545-3253 
de Masa Corporal (IMC) promedio de 23,2 $\pm 2,16$ peso $\mathrm{kg} / \mathrm{talla}^{2}$. La prevalencia de sobrepeso (IMC $\geq 25$ ) en la muestra fue de 19,3\% ( $\mathrm{n}=26$ ), con el 80,1 \% ( $\mathrm{n}=109$ ) de los participantes con peso normal, según los criterios de la Organización Mundial de la Salud. Asimismo, IMC fue similar en los alumnos de las tres escuelas de formación: Esmic 23,65 $\pm 2,3 \mathrm{~kg} / \mathrm{m}^{2}$; Emsub 23,41 $\pm 2,4 \mathrm{~kg} / \mathrm{m}^{2}$; Espro 22,57 $\pm 1,4 \mathrm{~kg} /$ $\mathrm{m}^{2}$ ), el valor absoluto de masa grasa en los alumnos de la Espro evidenció un menor peso en grasa en comparación con los alumnos de la Esmic y la Emsub: Esmic 12,16 \pm

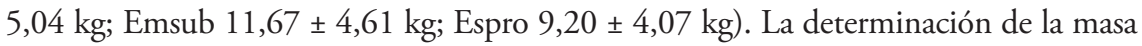
muscular esquelética tuvo diferencias de las dos escuelas con respecto a la Escuela Militar de Cadetes: Esmic 28,52 \pm 2,79 kg; Emsub 25,82 \pm 2,85 kg; Espro 26,83 $\pm 2,88 \mathrm{~kg}$ ). Conclusiones: estas diferencias en la composición corporal de los alumnos de las tres escuelas se deberían a factores como la carga, intensidad, frecuencia y duración en los tiempos de entrenamiento de cada una de las instituciones militares.

Palabras clave: composición corporal; impedancia eléctrica; personal militar.

\section{Introducción}

El personal militar debe cumplir una serie de tareas específicas en los diferentes ambientes de los teatros de operaciones y que se consideran factores estresores por diferentes causas, como pueden ser el déficit calórico, la privación del sueño, alteraciones del estado ánimo y la actividad física continua, llegando hasta casos de fatiga (1). El desarrollo de las operaciones militares le exige al soldado altos niveles de capacidad aeróbica, de reserva energética, así como de fuerza muscular, de manera que un buen fitness (entendido como resistencia aeróbica, resistencia muscular, fuerza, flexibilidad y buena composición corporal) y en general un adecuado acondicionamiento físico son factores importantes en su desempeńo y en la prevención de lesiones osteomusculares (2). No obstante que la actividad física es esencial para mejorar el acondicionamiento físico y prevenir la obesidad (3), durante las últimas décadas el fitness y la capacidad aeróbica han disminuido en la gente joven de los países occidentales y ha aumentado la obesidad, tanto en personal civil como militar. En Colombia, esta situación constituye actualmente un gran reto para el entrenamiento militar, sobre todo si se tiene en 
cuenta que ciertas actividades físicas se han reducido debido a los desarrollos logísticos y tecnológicos (4).

De igual forma, las tasas de obesidad están en aumento en todo el mundo, a tal punto que actualmente se considera, después del consumo de cigarrillo, como una condición epidémica involucrada en el aumento de las tasas de morbilidad y mortalidad por causas prevenibles relacionadas con la salud. Por esta razón, la prevención y las estrategias para controlar esta patología deben iniciar en la infancia y la adolescencia para prevenir la obesidad del adulto. Adicionalmente, se ha reportado que los adultos que fueron obesos durante la niñez tienen mayor riesgo de morbi-mortalidad, independientemente de su peso en la edad adulta (5).

Específicamente en el ámbito militar, el uso del peso y la talla como parámetros para determinar la incorporación de sujetos con problemas de desnutrición crónica o peso inadecuado para la talla ha dado un giro radical, ya que actualmente estas variables se emplean sobre todo para prevenir el sobrepeso y la obesidad del personal que se va a incorporar. Así mismo, los determinantes de la composición corporal se valoran actualmente más en función de asegurar una capacidad física aceptable y un rápido desarrollo de la fuerza a través de una adecuada nutrición y actividad física regular (6); inclusive, en algunos casos se consideran como factores predictores del rendimiento en la fuerza (7). Cabe anotar, sin embargo, aunque los índices de obesidad en militares están por debajo de los promedios de la población en general, una composición corporal inadecuada impacta negativamente los costos en los servicios de salud. Esto reafirma la necesidad de crear, fomentar e implementar políticas y programas que busquen reducir la prevalencia del sobrepeso y la obesidad (8).

En este contexto, valorar la composición corporal es un requisito indispensable para monitorear los tipos de obesidad, el estado nutricional, los objetivos del entrenamiento y, en general, el estado de salud (9). Se debe tener en cuenta que el exceso de tejido adiposo actúa como peso muerto en actividades en las cuales la masa del cuerpo debe vencer repetidamente la gravedad durante la locomoción o el salto, es decir, disminuye el rendimiento militar y deportivo e incrementa la demanda energética de la acti- 
vidad. En contraste, la masa libre de grasa contribuye a la producción de energía durante actividades de alta intensidad, por lo cual mejora la fuerza absoluta y la resistencia a cargas estáticas y dinámicas (10). Por estas características, la masa grasa y la masa libre de grasa (magra) son los componentes más usados para identificar el tipo de requerimientos nutricionales y los gastos energéticos del organismo (11).

Los expertos en nutrición deportiva usan los datos obtenidos de la composición corporal para desarrollar intervenciones específicas de tipo dietario, así como para orientar a los entrenadores y preparadores físicos para optimizar y evaluar los programas de entrenamiento deportivo. De igual forma, la evaluación de la composición corporal se usa con frecuencia en los campos del entrenamiento físico militar para evaluar la efectividad de los programas de los individuos que quieren bajar de peso o comenzar un entrenamiento funcional (fuerza, resistencia, definición de la figura corporal) y/o deportivo (12). En este sentido, el control del peso corporal en los diferentes tipos de entrenamiento es uno de los problemas clave durante la actividad militar y deportiva, pues no sorprende que algunos militares y atletas tiendan a aumentar y acelerar sus rutinas de acondicionamiento físico, lo cual puede tener un efecto negativo para su salud o en su desempeño en el área de operaciones o en competencia. Por lo tanto, es importante mejorar la eficiencia tecnológica en los sistemas de entrenamiento militar y deportivo, sobre todo respeto al control de los procesos que lleven a optimizar los resultados deseados.

En los últimos años el análisis por bioimpedancia (BIA, por su sigla en inglés) está siendo usado ampliamente en la práctica médica deportiva y se están desarrollando más y mejores métodos para determinar la estructura y los componentes del cuerpo (13). La bioimpedancia tiene como principio administrar al sujeto un flujo de corriente eléctrica alterna de una o más radiofrecuencias, transmitida por un electrodo adherido a la superficie de la piel, con el fin de caracterizar los tejidos que tengan o no buena conducción, lo mismo que de los fluidos que componen el cuerpo (14), ya que los flujos de corrientes en diferentes velocidades dependen de la composición del cuerpo. Como se sabe, el agua es buena conductora de la corriente, lo mismo que los tejidos ricos en electrolitos como el músculo, mientras que 
el tejido graso, óseo y los espacios corporales neumáticos son malos conductores de la corriente (15).

Con el fin de aclarar los fundamentos de la bioimpedancia, a continuación, se definen brevemente los conceptos básicos de esta técnica. En primer lugar, la impedancia $(Z)$ es la frecuencia dependiente de la oposición al flujo de corriente por parte del conductor (por ejemplo, el cuerpo). Geométricamente, la impedancia es el vector compuesto de dos parámetros dependientes de frecuencias (la resistencia $\mathrm{R} y$ la reactancia $\mathrm{Xc}$ ). La resistencia es la oposición al flujo de corriente cuando pasa a través del cuerpo y la reactancia es la demora en la conducción eléctrica causada por las membranas celulares, las interfaces de los tejidos y las sustancias no-iónicas. En segundo lugar, la capacitancia es una función de la reactancia, que se aumenta cuando las membranas celulares almacenan una porción de la corriente en un espacio de tiempo. Ese almacenamiento temporal de las cargas de corriente crea una desviación de la fase o ángulo de fase (AF), cuantificada como la relación de la Arco tangente de la reactancia y la resistencia expresada en grados (Xc / $\mathrm{R}) \mathrm{x}\left(180^{\circ} / \pi\right)(15)$. El ángulo de fase es una medida proporcionada directamente por los equipos de bioimpedancia y se usa como un marcador de la integridad de la membrana celular y de la masa celular del cuerpo, además de ser un predictor de morbi-mortalidad de enfermedades de tipo crónicas, como pueden ser la falla renal (16), la insuficiencia cardiaca congestiva (17), patologías oncológicas (18) o desnutrición (19).

En tercer lugar, el ángulo de fase (AF) es la relación del cálculo entre la resistencia $(\mathrm{R})$ de los tejidos al paso de la corriente (que depende del estado de hidratación) y la reactancia (Xc) de los tejidos asociados a la celularidad, el tamaño de las células y la integridad de la membrana celular. Un ángulo de fase disminuido es consistente con muerte celular o una alteración en la selectividad de la membrana celular, mientras que valores aumentados se asocian con integridad y vitalidad de las membranas celulares y el cuerpo de masa celular. En población sana, el sexo, la edad y el Índice de Masa Corporal son los principales determinantes del ángulo de fase (20). Por su parte, en personas saludables los rangos normales del ángulo de fase oscilan entre $5^{\circ}$ y $7^{\circ}$, y en atletas pueden alcanzar ángulos de fase hasta $9,5^{\circ}$. No obstante, 
la información disponible, en el medio militar se encontró escasa literatura sobre el uso de la BIA para determinar la composición en ciertos grupos de formación militar y mucho menos en Colombia. Por esta razón, el presente estudio hace una caracterización general de los parámetros bioeléctricos de la composición corporal de alumnos de último nivel de formación militar en las escuelas de formación del Ejército Nacional de Colombia.

\section{Metodología}

El diseño del estudio fue observacional, descriptivo, transversal con componente analítico, en el cual se valoró la composición corporal por BIA de 135 estudiantes de último nivel de instrucción militar de las tres escuelas de formación del Ejército Nacional. El estudio fue realizado en tres centros de formación militar colombiano: 1) Escuela Militar de Cadetes "General José María Córdova” (Esmic), Bogotá, D. C., donde se forman los oficiales del Ejército Nacional. 2) Escuela de Suboficiales Sargento Inocencio Chincá (Emsub), Melgar (Tolima). 3) Escuela de Soldados Profesionales Pedro Pascasio Martínez Rojas (Espro), Nilo (Cundinamarca). Este trabajo fue aprobado por el Comité de Ética de Ciencias Sociales y Exactas (Cecse) de la Esmic, según acta 4363 REG-AL-FOL-71/02-2018. Los sujetos del estudio participaron en una reunión antes de tomar los datos para explicarles los objetivos y la metodología de la investigación. Allí no solo tuvieron la oportunidad de obtener toda la información, sino que se les aseguró la confidene cialidad de los datos con la firma de un consentimiento informado. Durante todo el estudio, los participantes permanecieron anonimizados y en los casos en que algún sujeto se retiró de la investigación no hubo repercusión en su carrera militar.

Las variables de la composición corporal fueron tomadas por una nutricionista-antropometrista categoría ISAK 1 (International Society for the Advancement of Kinanthropometry), de acuerdo con el protocolo pretest, donde además se especifica que los datos se deben tomar en horas de la mañana y a la misma hora para todos los grupos. Los sujetos fueron pesados en ropa interior, sin zapatos, sin que hubieran realizado ejercicio físico las veinticuatro 
horas previas al análisis, sin que hubieran ingerido alimentos cuatro horas antes de la prueba, con un buen estado de hidratación, así como haber realizado su última micción treinta minutos antes de comenzar las pruebas.

Para medir la composición corporal por bioimpedancia eléctrica se usó el equipo SECA mBCA 525 (Medical Body Composition Analyzer, Hans E. Ruth S.A, Hamburg, Germany), que usa el método de medición de análisis de impedancia bioeléctrica de ocho puntos, con diecinueve frecuencias de medición que van desde 1 hasta $1.000 \mathrm{kHz}$, y con valores de medición de impedancia $(Z)$, resistencia $(R)$, reactancia $\left(X_{c}\right)$ y ángulo de fase $(\Phi)$. Además, el equipo tiene un área de medición de impedancia de $10 \Omega$ a $1.000 \Omega$ y medición de corriente de $100 \mu \mathrm{A}$, lo cual permite hacer el registro multisegg mento del cuerpo y el ángulo de fase $\left(0^{\circ}-20^{\circ}\right)$. La talla fue tomada usando un estadiómetro de plataforma manual (Seca 274, Hamburg, Germany). La circunferencia de cintura se midió en el punto medio entre la última costilla y la cresta iliaca usando una cinta métrica (Ohaus ${ }^{\oplus}-8004 \mathrm{MA}$, Parsippany, NJ, USA). Los datos obtenidos fueron analizados y almacenados mediante el software Seca Analytics $115^{\circ}$.

\section{Análisis estadístico}

Para analizar los datos se determinaron medidas de tendencia central (medias y medianas), medidas de dispersión (desviaciones estándar, error absoluto de la desviación estándar, límite superior e inferior del intervalo de confianza del $95 \%$ ). Asimismo, se valoró la normalidad de distribución de los datos mediante las pruebas de Kolmogorov-Smirnov y se realizó un análisis de varianza de un factor (Anova) con pruebas post hoc (Bonferroni y Games-Howell) para comparaciones múltiples, con el fin de determinar las diferencias entre las variables de composición corporal en los participantes del estudio en cada una de las escuelas de formación, teniendo en cuenta los supuestos para la realización de la prueba. También se utilizó estadística no paramétrica mediante la prueba de Kruskal Wallis en el tipo de datos que así lo requirieron y se determinó la correlación entre el IMC y las variables de composición corporal de la muestra mediante la prueba de Spearman. 
Finalmente, el análisis de los datos se realizó con el software estadístico Statistical Package for the Social Sciences ${ }^{\circledR}$ V.24 (SPSS 24) y Graph Pad Prism 7 para diagramar los resultados. El nivel de significancia estadística se definió por una confianza del $95 \%$ y el valor de la probabilidad menor de $\mathrm{p} \leq 0.05$.

\section{Resultados}

La muestra estuvo conformada por 135 cadetes de sexo masculino de las tres escuelas de formación militar del Ejército Nacional de Colombia que pertenecían al último nivel de formación militar: en el caso de la Esmic, de octavo semestre; en el de la Emsub, de tercer semestre de formación militar, y en el de la Espro, posterior a los seis meses de la fase de entrenamiento militar básico. Las edades de los estudiantes oscilaron entre los 18 a 25 ańos ( $21 \pm 1,5$ años), con un peso de 66,3 \pm 7,9 kg y un Índice de Masa Corporal promedio de 23,2 $\pm 2,16$ talla $^{2} /$ peso, con una talla promedio de 1,68 \pm 0,07 m. La determinación del perímetro de cintura para el análisis de bioimpedancia arrojó $0,786 \pm 0,056 \mathrm{~m}$. La prevalencia de sobrepeso (IMC $\geq 25$ ) en la muestra fue de 19,3\% ( $\mathrm{n}=26)$, con el 80,1\% ( $\mathrm{n}=109)$ de los participantes con peso normal, según los criterios de la Organización Mundial de la Salud (OMS).

Una vez realizado el análisis de la composición corporal, las características antropométricas y las variables bioeléctricas establecieron que el Índice de Masa Corporal (IMC) presentó valores muy parecidos en los estudiantes de las tres escuelas de formación: Esmic, 23,65 $\pm 2,3 \mathrm{~kg} / \mathrm{m}^{2}$; Emsub, 23,41 $\pm 2,4 \mathrm{~kg} / \mathrm{m}^{2}$; Espro $22,57 \pm 1,4 \mathrm{~kg} / \mathrm{m}^{2}$. Los datos obtenidos en valor absoluto de masa grasa muestran que los alumnos de la Espro tienen un volumen de grasa significativamente menor en comparación con los alumnos de la Esmic y la Emsub: Esmic, 12,16 \pm 5,04 kg; Emsub, 11,67 \pm 4,61 kg; Espro, $9,20 \pm 4,07 \mathrm{~kg}$. En cuanto a la masa libre de grasa, se presentaron valores similares en la Esmic y la Espro, mientras que en la Emsub el valor fue significativamente menor: Esmic, 57,68 $\pm 5,31 \mathrm{~kg}$; Emsub, 52,54 $\pm 5,23 \mathrm{~kg}$; Espro, 56,25 $\pm 5,77 \mathrm{~kg}$. 
La determinación de la masa muscular esquelética varió en las dos escuelas con respecto a la Esmic (28,52 $\pm 2,79 \mathrm{~kg})$ : Emsub, 25,82 $\pm 2,85 \mathrm{~kg}$ y Espro, 26,83 $\pm 2,88 \mathrm{~kg}$. Esto se ve reflejado en el cálculo de las kilocalorías dadas por el gasto energético total de los individuos: Esmic, 3138,73 \pm $247,79 \mathrm{kcal}$; Emsub, 3018,05 $\pm 207,87 \mathrm{kcal}$; Espro, 3005,55 $\pm 164,74 \mathrm{kcal}$. Por último, en el análisis de los valores del ángulo de fase se evidenció que los alumnos de la Escuela de Suboficiales presentaron menor ángulo de fase en comparación con los de la Escuela de Cadetes y los de la Escuela de Soldados

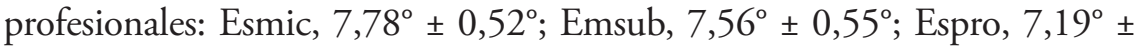
$0,66^{\circ}$. Sin embargo, cabe aclarar que estos datos están dentro de los valores normales para la población joven y activa físicamente (tabla 1).

Tabla 1. Variables de la composición corporal por bioimpedancia de los alumnos de las tres escuelas de formación del Ejército Nacional.

\begin{tabular}{lrrrrrr}
\hline \multirow{2}{*}{ Variable } & \multicolumn{2}{c}{ Esmic } & \multicolumn{2}{c}{ Emsub } & \multicolumn{2}{c}{ Espro } \\
\cline { 2 - 7 } & \multicolumn{1}{c}{$\mathbf{x}^{*}$} & \multicolumn{1}{c}{$\mathbf{d s} \boldsymbol{s}^{* *}$} & \multicolumn{1}{c}{$\mathbf{x}$} & \multicolumn{1}{c}{$\mathbf{d s}$} & \multicolumn{1}{c}{$\mathbf{x}$} & \multicolumn{1}{c}{$\mathbf{d s}$} \\
\hline Edad (ańos) & 22,58 & 1,234 & 21,38 & 1,775 & 21,24 & 1,26 \\
Peso (kg) & 69,846 & 8,667 & 64,220 & 7,7595 & 64,9244 & 6,0784 \\
Talla (m) & 1,7173 &, 06340 & 1,6553 &, 06244 & 1,6953 &, 07288 \\
IMC (kg/m²) & 23,651 & 2,3576 & 23,411 & 2,4618 & 22,578 & 1,34137 \\
Perímetro de la Cintura (cm) & 0,8078 & 0,0613 & 0,7902 & 0,0594 & 0,7609 & 0,0346 \\
Masa Grasa (kg) & 12,1633 & 5,04370 & 11,6736 & 4,6163 & 8,6667 & 2,5304 \\
Masa Libre de Grasa (kg) & 57,6833 & 5,3164 & 52,5464 & 5,2335 & 56,2578 & 5,7722 \\
Músculo Esquelético (kg) & 28,5216 & 2,7909 & 25,8207 & 2,8538 & 26,8389 & 2,8802 \\
Agua Corporal Total (kg) & 41,8756 & 4,0355 & 38,0689 & 3,9346 & 40,8978 & 4,3203 \\
Agua Extracelular (kg) & 16,3311 & 1,8115 & 15,1467 & 1,7238 & 16,6756 & 2,0061 \\
Gasto Energético (Kcal) & 3138,73 & 247,791 & 3018,05 & 207,875 & 3005,55 & 164,746 \\
Gasto en Reposo (Kcal) & 1739,83 & 130,337 & 1657,24 & 114,965 & 1669.75 & 91,526 \\
Ángulo de Fase (o) & 7,78 &, 522 & 7,56 &, 553 & 7,19 &, 6643 \\
\hline
\end{tabular}

${ }^{*}$ Media (x).

** Desviaciones estándar (ds)

Fuente: Original de los autores.

En el set de gráficas se presenta las comparaciones de algunas variables de la composición corporal entre las tres escuelas, para lo cual se realizó 
un análisis de varianzas de un factor (Anova) con pruebas para múltiples comparaciones de Bonferroni o Games Howell, según el caso, para datos que presentaron normalidad en su distribución. También se realizó la comparación intergrupos para datos con distribución no paramétrica con el estadístico de Kruskal Wallis, donde se encontraron diferencias estadísticamente significativas en las siguientes variables: en el IMC entre la Esmic y la Espro ( $p$ $=0,029$ ) (figura 1). En la cantidad de masa grasa entre la Esmic y la Espro $(p=0,0001)$ y entre la Emsub y la Espro $(p=0,001)$ (figura 2). En la masa muscular esquelética entre la Esmic y la Emsub $(p=0,0001)$ y entre la Esmic y la Espro $(p=0,0001)$ (figura 3). En la masa libre de grasa entre la Esmic y la Emsub $(p=0,0001)$ y entre la Emsub y la Espro $(p=0,005)$ (figura 4 ). En el gasto energético total entre la Esmic y la Emsub $(p=0,021)$ y entre la Esmic y la Espro $(p=0,009)$ (figura 5); lo mismo que en el ángulo de fase, donde se presentaron diferencias estadísticamente significativas entre la Esmic y la Emsub $(p=0,0001)$ y entre la Emsub y la Espro $(p=0,009)$ (figura 6 ). $\mathrm{El}$ análisis de correlación entre el IMC y las variables bioeléctricas evidenció relaciones significativas con el valor de la masa grasa $(r=0,83 ; p=0,0001)$ (figura 7) y con la masa muscular esquelética $(r=0,45 ; p=0,0001)$ (figura 8 ).

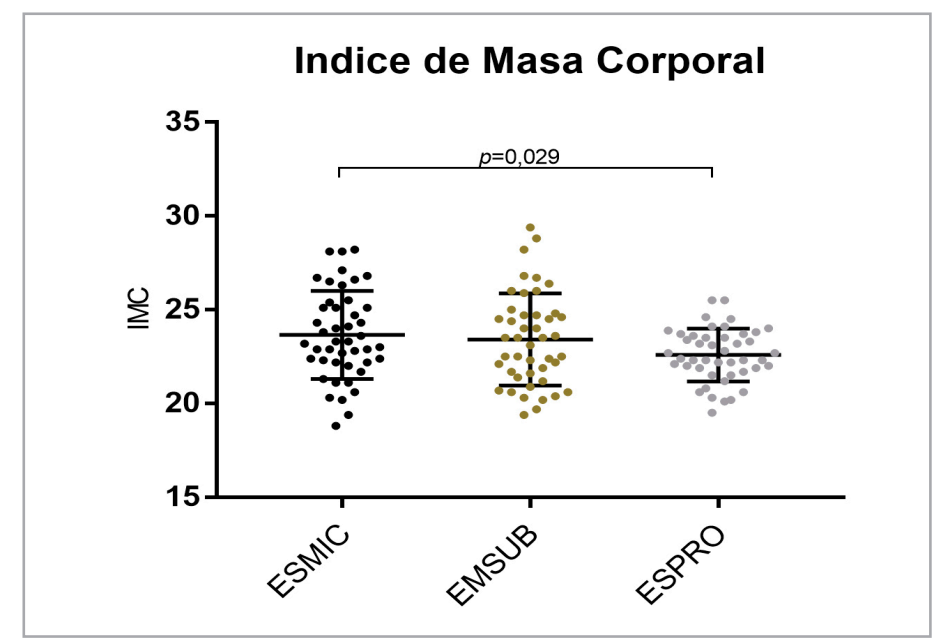

Figura 1. Comparación del Índice de Masa Corporal (IMC) entre los alumnos de último nivel de las tres escuelas de formación (Esmic, Emsub y Espro). Análisis de varianza (Anova) de comparación múltiple, estadístico de prueba Games-Howell. Fuente: Original de los autores. 


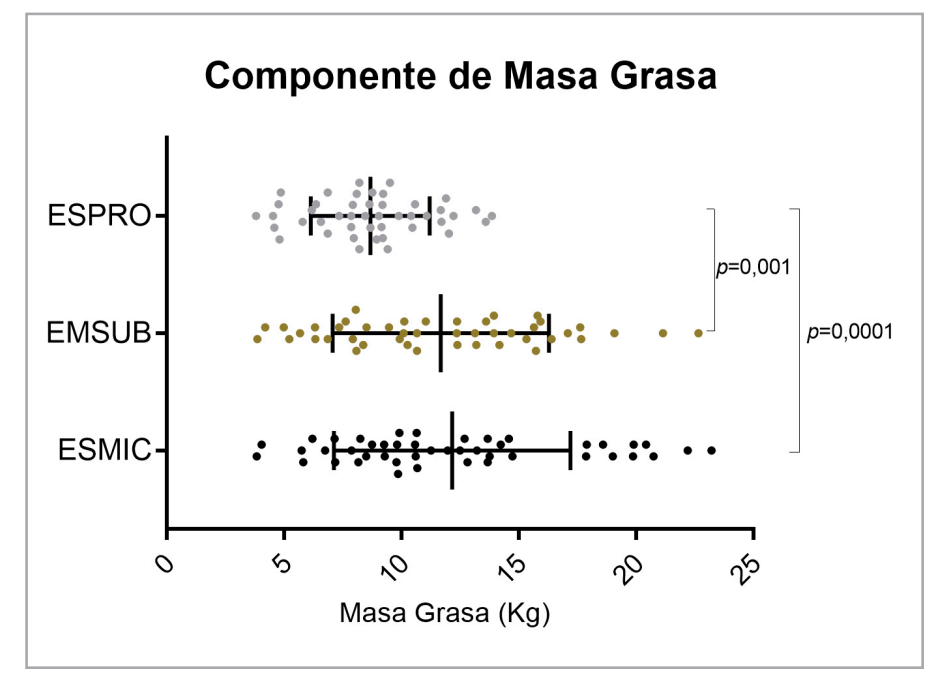

Figura 2. Comparación del componente de masa grasa en kilogramos entre los alumnos de último nivel de las tres escuelas de formación: Esmic, Emsub y Espro. Análisis de varianza (Anova) de comparación múltiple, estadístico de Games-Howell.

Fuente: Original de los autores.

\section{Componente de Masa Muscular Esqueletica}

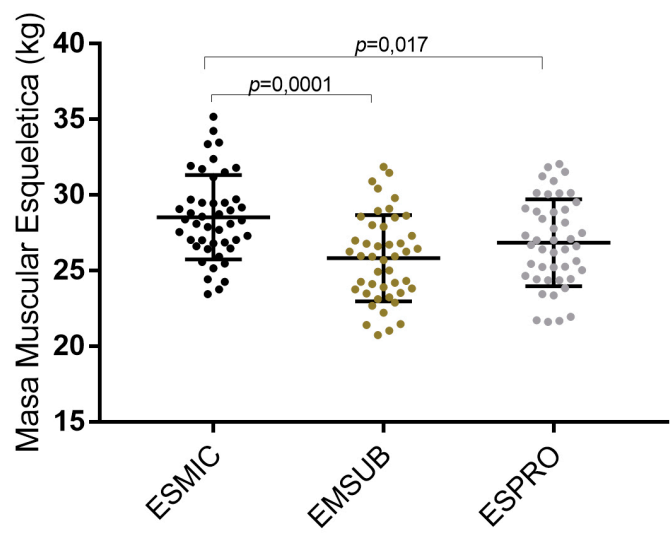

Figura 3. Comparación del componente de masa muscular esquelética en kilogramos entre los alumnos de último nivel de las tres escuelas de formación: Esmic, Emsub y Espro. Análisis de varianza (Anova) de comparación múltiple, estadístico de Bonferroni. Fuente: Original de los autores. 


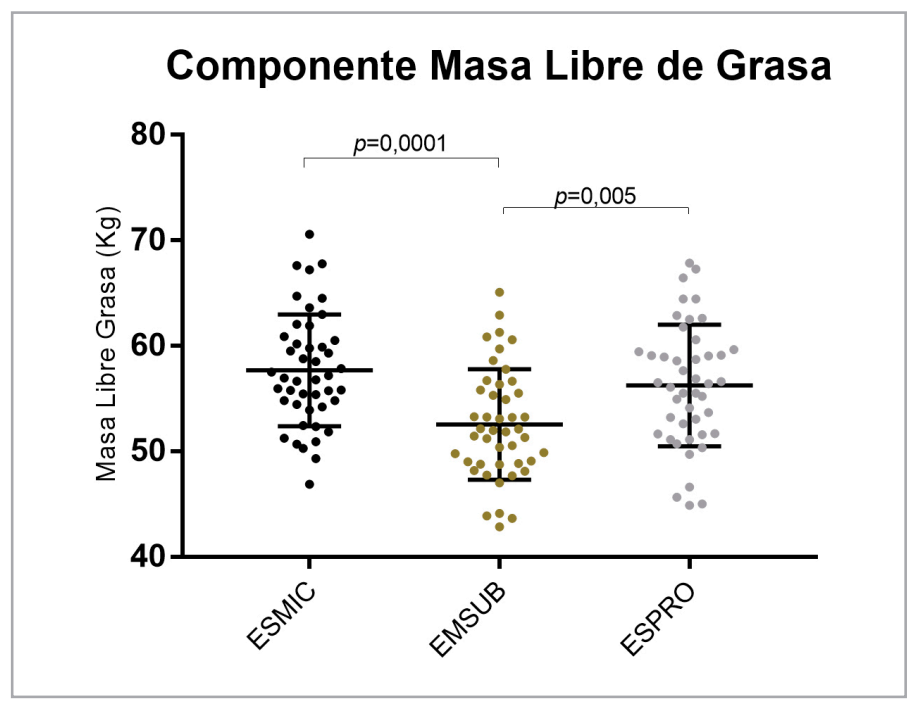

Figura 4. Comparación del componente de masa libre de grasa en kilogramos entre los alumnos de último nivel de las tres escuelas de formación: Esmic, Emsub y Espro. Análisis de varianza (Anova) de comparación múltiple, estadístico de Bonferroni.

Fuente: Material propio de los autores

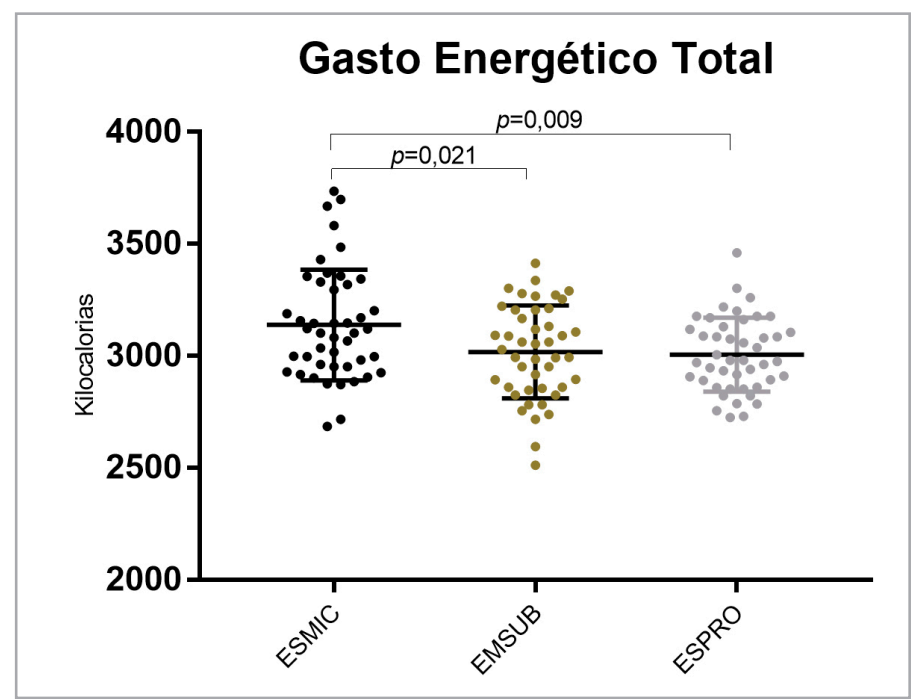

Figura 5. Comparación del gasto energético total en kilocalorías entre los alumnos de último nivel de las tres escuelas de formación: Esmic, Emsub y Espro. Análisis de varianza (Anova) de comparación múltiple, estadístico de Bonferroni.

Fuente: Original de los autores. 


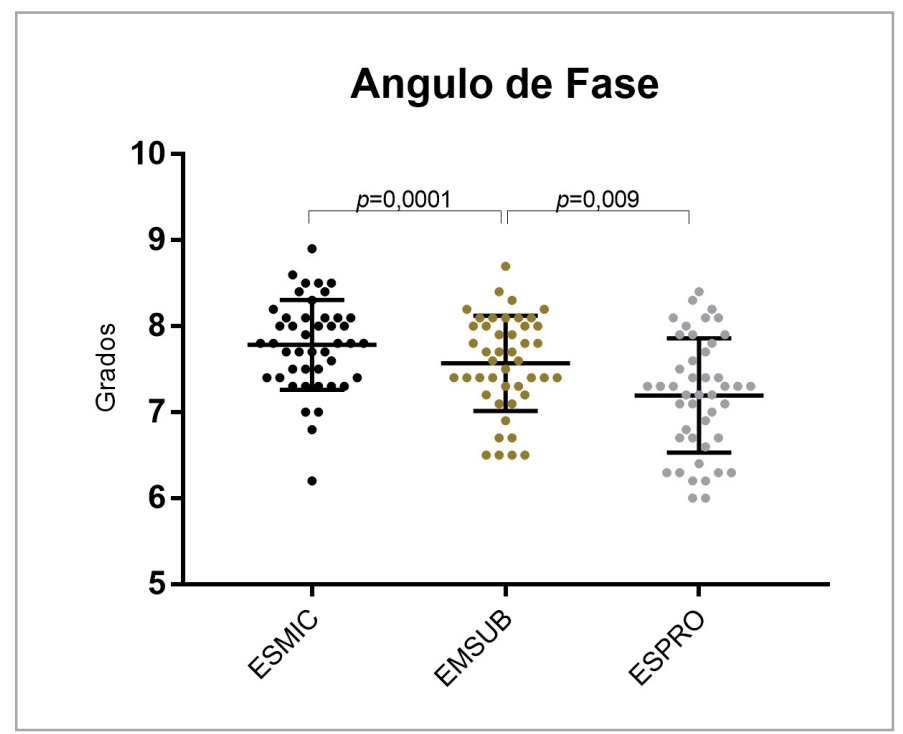

Figura 6. Comparación del ángulo de fase entre los alumnos de último nivel de las tres escuelas de formación Esmic, Emsub y Espro. Análisis de varianza (Anova) de comparación múltiple, estadístico de Bonferroni.

Fuente: Original de los autores.

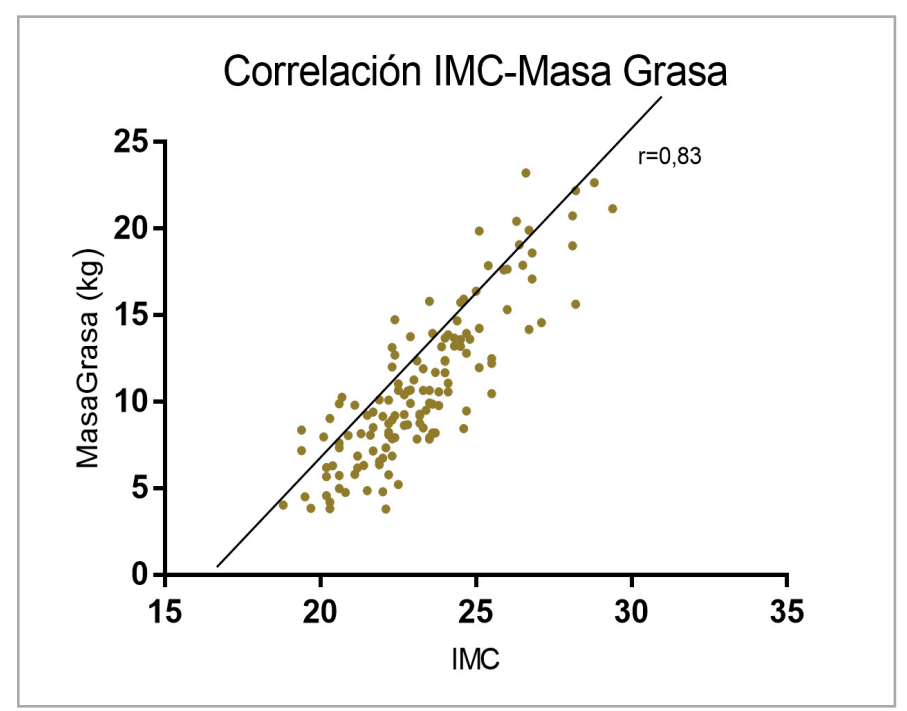

Figura 7. Correlación entre el IMC y la masa grasa del total de los participantes del estudio en las tres escuelas de formación del Ejército Nacional. Correlación de Spearman $(r=0,83)$. Fuente: Original de los autores. 


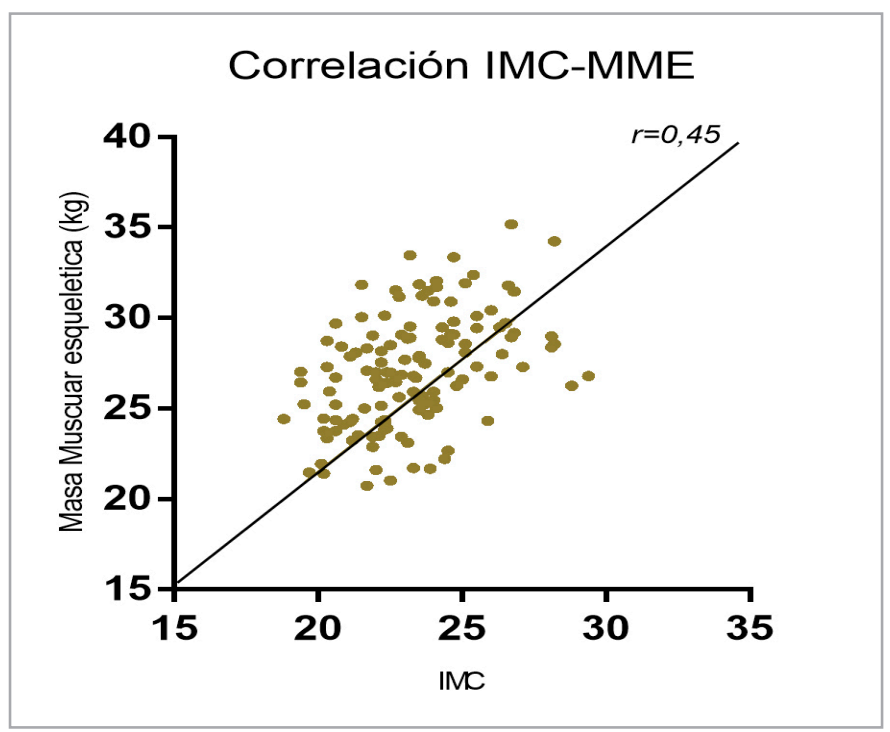

Figura 8. Correlación entre el IMC y la masa muscular esquelética de los participantes del estudio en las tres escuelas de formación del Ejército Nacional. Correlación de Spearman $(r=0,45)$.

Fuente: Original de los autores.

\section{Discusión}

El principal propósito de mantener adecuados niveles de fitness y composición corporal en el personal militar es que se consideran un criterio de selección para desempeñar labores propias de las demandas físicas del servicio militar, pues se asume que un adecuado peso corporal se correlaciona con un buen estado de salud, con una condición física y mental adecuada, lo mismo que de una apariencia militar adecuada (21). Los avances en los servicios de salud, en los desarrollos tecnológicos e informáticos, así como la mejoría de los requerimientos nutricionales en recientes décadas han llevado a un aumento en el promedio de peso, talla y masa libre de grasa de la población en general. Sin embargo, el riesgo de consumo excesivo de alimentos, tanto en cantidad como en calidad, lo mismo que la inactividad física, se han convertido en una preocupación institucional debido al impacto que tiene el sobrepeso en la salud y el desempeño del militar. Por esta razón, una de 
las preguntas más relevantes que se plantean las organizaciones encargadas de supervisar los planes de entrenamiento físico y militar es cómo evaluar el desempeño físico (fitness) del integrante de un ejército, frente a la cual se proponen cuatro componentes: la capacidad aeróbica, la potencia muscular, la resistencia y la composición corporal (22).

No obstante, hasta el momento no se había evaluado en conjunto las cualidades propias de la composición corporal del militar colombiano que cursa su entrenamiento técnico y táctico en las escuelas de formación del Ejercito Nacional. Precisamente, el aporte de este trabajo es que permite contrastar la caracterización física de esta población con estudios en otros países, lo mismo que entre a las tres escuelas, como se presenta a continuación.

El IMC — un indicador de cribado poblacional para detectar sobrepeso y obesidad que analiza la relación entre el peso y la talla - de toda la muestra estudiada fue 23,21 $\pm 2,16$ peso $\mathrm{kg} / \mathrm{talla}^{2}$. En contraste, Maldonado y cols. encontraron en 2017 valores similares del IMC de 22,7 $\pm 2,7$ peso $\mathrm{kg} / \mathrm{talla}^{2}$ en un estudio de 153 alumnos de escuelas de formación de soldados del Ejército en Ecuador, pero con la diferencia de que los datos de la composición corporal fueron obtenidos por pliegues cutáneos (23). En 2018, otro estudio de Cortés y cols. encontraron valores de IMC de 24,4 $\pm 2,22$ peso $\mathrm{kg} /$ talla $^{2}$ en 72 cadetes que asistieron a un curso de combate de doce semanas en Colombia; estos valores fueron recolectados por bioimpedancia eléctrica antes de que los soldados asistieran al entrenamiento del Curso de Combate (24). En 2017, Duran-Agüero y cols. hallaron en una muestra de 415 soldados de una brigada chilena de paracaidismo militar que el valor de IMC en militares menores de 30 años era de 25,4 $\pm 2,9$ peso $\mathrm{kg} /$ talla $^{2}$ (25). Por su parte, en 2016 Gómez y cols. determinaron por bioimpedancia un IMC de $26,90 \pm 0,80$ peso $\mathrm{kg} / \mathrm{talla}^{2}$ en 288 militares mexicanos (26). Finalmente, en estudios realizados en el Reino Unido, Blacker y cols. evaluaron en 2013 diferentes parámetros de 119 integrantes de distintos componentes de las fuerzas militares (Armada, Fuerza Área y Ejército) y encontraron que los integrantes del Ejército tenían un IMC de 22,2 $\pm 2,6$ peso kg/ talla ${ }^{2}$ (27). Como se observa, el promedio general del IMC en los diferentes ejércitos evaluados 
no tiene mayores diferencias, con excepción de la población chilena, donde se valoraron sujetos con edades superiores a los 25 años, lo cual se relaciona con el aumento del IMC asociado a las condiciones de edad.

En cuanto a la diferencia del IMC entre las tres escuelas de formación del Ejército de Colombia, se encontraron diferencias significativas entre los alumnos de la Esmic respecto a los de la Espro. Este hallazgo está íntimamente relacionado con menores valores en el componente de masa grasa en la Espro, lo cual se explica por varias razones: en primer lugar, tienen un entrenamiento más corto en duración, pero con mayor intensidad (doce semanas de entrenamiento constante con carga permanente de equipo de campaña); en segundo lugar, la altura de la zona donde se hace el entrenamiento - 336 metros sobre el nivel del mar, mientras que en Bogotá es de 2.600 m. s. n. m. - hace que las pérdida sensible e insensible de agua sea mayor en la Espro. Considerando estas condiciones y para evitar los sesgos de medición, se aplicó el protocolo pretest de bioimpedancia, con lo cual no se afectó la valoración del peso y el componente de masa grasa, pues al ser menor en esta población, se reflejaba directamente con un menor IMC.

Asimismo, el componente de masa grasa se estimó en 10,83 \pm 4,45 kg. Para esta misma variable, estudios similares como los de Cortés y cols. en 2018 encontraron valores de 6,27 \pm 4,88 kg. En el que realizaron DuranAgüero y cols. en Chile en 2017 los valores de masa grasa fueron de 16,1 \pm 5,6 kg, mientras que Vásquez Guzmán y cols. en México en 2016 reporta 19,32 $\pm 3,07 \mathrm{~kg}$. Al observar estas últimas poblaciones, los valores de IMC mayores se podrían atribuir al aumento de la masa grasa, así como a problemas de sobrepeso relacionado con la edad y bajo nivel de actividad física. En cuanto a la comparación entre las tres escuelas, la Espro presentó menor cantidad de masa grasa en comparación con las otras dos escuelas de formación del Ejército Nacional.

Por su parte, el componente de masa libre de grasa se determinó para las tres escuelas con una media de 55,49 $\pm 5,82 \mathrm{~kg}$. En un estudio previo en militares colombianos realizado por Cortés y cols. en 2018 se encontraron valores de 66,3 $\pm 6,12 \mathrm{~kg}$ para su muestra (24). Mientras que en militares 
chilenos la masa libre de grasa fue de 57,8 $\pm 6,0 \mathrm{~kg}$. Por último, en la pobla-

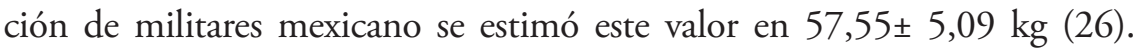
Asimismo, en la comparación entre las escuelas de formación se hallaron diferencias significativas entre los valores de la masa libre de grasa, con la muestra de la Emsub como la que presenta menores componentes de tejido libre de grasa. Cabe anotar que en la asociación de este componente con el IMC no se encontró una correlación fuerte entre estas dos variables $(r=$ $0,33 ; p=0,001)$, lo cual coincide con los valores obtenidos en población militar mexicana $(r=0,33)$ y muy diferente a la correlación de los componentes en población chilena $(r=0,61)$.

En cuanto al promedio del componente de masa muscular esquelética en la población de estudio se estableció un valor de 27,06 $\pm 3,03 \mathrm{~kg}$, el cual es disímil al que encontró Cortés y cols. en población similar: $63 \pm 5,09$ $\mathrm{kg}$ (24). La comparación entre las escuelas evidenció que los militares de la Emsub presentaron menor componente de músculo esquelético, con difep rencias significativas respecto a los militares de la Esmic y la Espro. Se debe señalar, además, que la correlación obtenida entre el IMC y la masa muscular esquelética es positiva entre estas dos variables $(p=0,45)$.

Ahora bien, a pesar de que se documentaron valores obtenidos de las variables de la composición corporal como gasto energético total, gasto energético en reposo, agua corporal total, agua extracelular y ángulo de fase, no se encontró literatura en militares para contrastar. Sin embargo, es importante contar con una línea de base que describa las características bioeléctricas de la composición corporal en este tipo de poblaciones. Particularmente, el estudio de estas variables arrojó diferencias significativas cuando se compararon los resultados obtenidos por las tres escuelas, diferencias que se podrían explicar en razón a tiempos, intensidad y frecuencia de los entrenamientos; no obstante, en términos generales, estos hallazgos no tienen variaciones significativas con respecto a los estándares reportados en otros estudios sobre población militar. 


\section{Conclusiones}

Aunque son muy pocos los estudios que analizan la composición corporal por bioimpedancia eléctrica en jóvenes y menos aun cuando se trata de una población militar, este trabajo determinó las variables de la composición corporal de mayor uso en nutrición deportiva y entrenamiento militar.

Este estudio piloto mostró ciertas diferencias estadísticas en los componentes de la composición corporal y variables bioeléctricas en relación con la escuela de formación. A pesar de que la muestra es homogénea, los resultados del estudio evidencian que en la Espro se presentan porcentajes de masa grasa menores a la Esmic y la Emsub. Esta diferencia se podría explicar por las características del entrenamiento intensivo y continuo en los cuatro meses de fase de instrucción, durante la permanencia del militar en la Escuela de Soldados Profesionales, mientras que en la Escuela de Suboficiales esta fase de instrucción y permanencia en la escuela es de año y medio y en la Escuela de Oficiales es de cuatro ańos. Caso contrario sucede con la masa muscular esquelética, que es mayor en la Esmic y Espro en comparación con la Emsub. Este es un primer paso para desarrollar estudios que permitan caracterizar mejor las variables de interés con muestras mucho más representativas de todas las unidades del Ejército Nacional.

\section{Agradecimientos}

Agradecemos a los directivos y personal integrante de la Escuela Militar de Cadetes "General José María Córdova”, de la Escuela de Suboficiales Sargento Inocencio Chincá y de la Escuela de Soldados Profesionales Pedro Pascasio Martínez Rojas por su apoyo incondicional en el desarrollo del presente estudio. Los autores declaramos no tener ningún tipo de conflicto de intereses en el desarrollo del presente estudio. 


\section{Financiación}

Este estudio fue realizado con recursos de la Convocatoria Interna 001-2017 del Comando de Apoyo Tecnológico de Ejército de Colombia, según Acta 65060 del 5 de julio de 2017.

\section{Referencias}

1. Task Group 019. Report RTOT. Optimizing Operational Physical Fitness (Optimisation de l' aptitude physique opérationnelle) [Internet]. 2009. 342 pp. Disponible en: http:// citeseerx.ist.psu.edu/viewdoc/download?doi=10.1.1.214.8364\&rep=rep1\&type=pdf

2. Santtila M, Keijo H, Laura K, Heikki K. Changes in cardiovascular performance during an 8-week military basic training period combined with added endurance or strength training. Mil Med. 2008; 173 (12): 1173-9.

3. Haskell WL, Lee IM, Pate RR, Powell KE, Blair SN, Franklin BA, et al. Physical activity and public health: updated recommendation for adults from the American College of Sports Medicine and the American Heart Association. Med Sci Sports Exerc. 2007; 39 (8): 1423-34.

4. Cawley J, Maclean JC. Unfit for Service: the implications of rising obesity for U.S. military recruitment. Health Econ. 2012 noviembre; 21 (11): 1348-66. DOI: http:// dx.doi.org/10.1002/hec.1794

5. Shi H, Jiang B, Wei Sim JD, Chum ZZ, Ali N Bin, Toh MH. Factors associated with obesity: a case-control study of young adult Singaporean males. Mil Med [Internet]. 2014; 179 (10): 1158-65. DOI: http://dx.doi.org/10.7205/MILMED-D-14-00064

6. Pihlainen K, Santtila M, Hakkinen Keiji KH. Associations of physical fitness and body composition characteristics with simulated military task performance. J Strength Cond Res. 2018 abril; 32 (4): 1089-98. DOI: http://dx.doi.org/10.1519/ JSC.0000000000001921.

7. Bahamondes-Avila C, Cárcamo-Oyarzún J, Aedo-Muñoz E, Rosas-Mancilla M. Relación entre indicadores antropométricos regionales de masa muscular y potencia de extremidades inferiores en deportistas juveniles de proyección. Rev Bras Ciências do Esporte. 2018; 40(3): 295-301.

8. Pierce JR, DeGroot DW, Grier TL, Hauret KG, Nindl BC, East WB, et al. Body mass index predicts selected physical fitness attributes but is not associated with performance on military relevant tasks in U.S. Army Soldiers. J Sci Med Sport [Internet]. 2017 noviembre; 20 (2017): S79-84. DOI: http://dx.doi.org/10.1016/j.jsams.2017.08.021

9. Durnin JV, Womersley J. Body fat assessed from total body density and its estimation from skinfold thickness: measurements on 481 men and women aged from 16 to 72 
year. Br J Nutr [Internet]. 1974 julio; 32(1): 77-97. DOI: http://dx.doi.org/10.1079/ bjn 19740060

10. Mala L, Maly T, Zahalka F, Bunc V, Kaplan A, Jebavy R, et al. Body composition of elite female players in five different sports games. J Hum Kinet. 2015 marzo; 45 (1): 207-15. DOI: http://dx.doi.org/10.1515/hukin-2015-0021

11. Nelson KM, Weinsier RL, Long CL, Schutz Y. Prediction of resting energy expenditure from fat-free mass and fat mass. Am J Clin Nutr [Internet]. 1992 noviembre [citado 2017 octubre 23]; 56(5): 848-56. DOI: http://dx.doi.org/10.1093/ajcn/56.5.848

12. Nickerson BS, Esco MR, Kliszczewicz BM, Freeborn TJ. Comparison of bioimpedance and underwater weighing body fat percentage before and acutely after exercise at varying intensities. J Strength Cond Res. 2017; 31(5):1395-402. DOI: http://dx.doi. org/10.1519/JSC.0000000000001716

13. Dopsaj M, Markovic M, Kasum G, Jovanovic S, Koropanovski N, Vukovic M, et al. Discrimination of different body structure indexes of elite athletes in combat sports measured by multi frequency bioimpedance method. Int J Morphol [Internet]. 2017 marzo; 35 (1): 199-207.

14. Portao J, Bescós R, Irurtia A, Cacciatori E, Vallejo L. Valoración de la grasa corporal en jóvenes físicamente activos: Antropometría vs bioimpedancia. Nutr Hosp. 2009 octubre; 24(5):529-34. Disponible en: http://scielo.isciii.es/scielo.php?script=sci_ arttext\&pid=S0212-16112009000500003

15. Mulasi U, Kuchnia AJ, Cole AJ, Earthman CP. Bioimpedance at the bedside: current applications, limitations, and opportunities. Nutr Clin Pract. 2015 abril; 30 (2): 180-93. DOI: http://dx.doi.org/10.1177/0884533614568155

16. Caravaca F, del Viejo CM, Villa J, Gallardo RM, Ferreira F. Estimación del estado de hidratación mediante bioimpedancia espectroscópica multifrecuencia en la enfermedad renal crónica avanzada. Nefrología. 2011 septiembre; 31 (5): 537-44. DOI: http:// dx.doi.org/10.3265/Nefrologia.pre2011.Apr.10936

17. Colín-Ramírez E, Castillo-Martínez L, Orea-Tejeda A, Vázquez-Durán M, Rodríguez $\mathrm{AE}$, Keirns-Davis C. Bioelectrical impedance phase angle as a prognostic marker in chronic heart failure. Nutrition. 2012 septiembre; 28(9): 901-5. DOI: http://dx.doi. org/10.1016/j.nut.2011.11.033

18. Gupta D, Lammersfeld CA, Vashi PG, King J, Dahlk SL, Grutsch JF, et al. Bioelectrical impedance phase angle as a prognostic indicator in breast cancer. BMC Cancer [Internet]. 2008 agosto; 8(1): 249. DOI: http://dx.doi.org/10.1186/1471-2407-8-249

19. Urbain P, Birlinger J, Ihorst G, Biesalski H-K, Finke J, Bertz H. Body mass index and bioelectrical impedance phase angle as potentially modifiable nutritional markers are independent risk factors for outcome in allogeneic hematopoietic cell transplantation. Ann Hematol [Internet]. 2013 enero; 92 (1): 111-9. DOI: http://dx.doi.org/10.1007/ s00277-012-1573-4 
20. Barrea L, Muscogiuri G, Macchia PE, Di Somma C, Falco A, Savanelli MC, et al. Mediterranean diet and phase angle in a sample of adult population: results of a pilot study. Nutrients. 2017 febrero; 9 (2) :1-14. DOI: http://dx.doi.org/10.3390/ nu9020151

21. Naghii MR. The importance of body weight and weight management for military personnel. Mil Med. 2006 junio; 171(6): 550-5. DOI: http://dx.doi.org/10.7205/ milmed.171.6.550

22. Sackett P, Anne, M. Physical fitness and musculoskeletal injury. En: National Research Council of the National Academics. Assessing fitness for military enlistment: physical, medical and mental health standards. Washington, DC: The National Academies Press; 2005. pp. 66-108. Disponible en: https://www.nap.edu/read/11511/chapter/1

23. Maldonado I, SC. Perfil antropométrico y composición corporal en aspirantes de la escuela de formación de soldados del ejército. Rev Cuba Investig Biomédicas. 2017 enero; 36(2): 1-15. Disponible en: http://www.revibiomedica.sld.cu/index.php/ibi/ article/view/3

24. Cortés Fernández S, Camargo IY, Botero Rosas D. Modificaciones en el índice de masa y composición corporal en personal activo del Ejército colombiano: un estudio de caso. Rev Cient Gen José María Córdova. 2018; 16 (22): 93-106. doi: http://dx.doi. org/10.21830/19006586.297

25. Durán-Agüero S, Maraboli Ulloa D, Fernández Frías F, Cubillos Schmied G. Composición corporal en soldados chilenos del Regimiento Buin. Revista Española Nutrición Humana y Dietética. 2017; 21 (1): 11.

26. Vázquez-Guzmán MA, Carrera-Rodríguez G, Durán-García AB G-OO. Correlación del índice de masa corporal con el índice de masa grasa para diagnosticar sobrepeso y obesidad en población militar. Rev Sanid Mil Mex. 2016 (6); 70 (6): 505-15.

27. Blacker SD, Horner FL, Brown PI, Linnane DM, Wilkinson DM, Wright A, et al. Health, fitness, and responses to military training of officer cadets in a gulf cooperation council country. Mil Med. 2013 diciembre; 176 (12): 1376-81. DOI: http://dx.doi. org/10.7205/milmed-d-11-00166 